\title{
Triterpenoids from Scorzonera veratrifolia Fenzl
}

\author{
Betül Çetin (D), Hasan Şahin (D), Aynur Sarı* (1) \\ Department of Pharmacognosy, Faculty of Pharmacy, Istanbul University, 34116, Istanbul, Turkey
}

Cite this article as: Çetin B, Sahin H, Sarı A. Triterpenoids from Scorzonera veratrifolia Fenzl. Istanbul J Pharm 48 (2): $23-27$.

\begin{abstract}
The genus Scorzonera (Asteraceae) has several species which are used as a vegetable and in folk medicine all around the world. A phytochemical study was carried out on petroleum ether fraction of methanol extract obtained from the roots of Scorzonera veratrifolia Fenzl (Bitlis / Turkey) to investigate the terpenoid composition of the plant. Column chromatography, preparative thin layer chromatography and gas chromatography (GC) were used for separation of the compounds. Their structures were determined by using GC-MS and NMR techniques. Seventeen triterpenoids ( $\beta$-amyrin, $\beta$-amyrin acetate, $\beta$-amyrinone, germanicol, germanicol acetate, germanicone, $\alpha$-amyrin, $\alpha$-amyrin acetate, $\alpha$-amyrinone, $\psi$ - taraxasterol, $\psi$ - taraxasterol acetate, taraxasterol, taraxasterol acetate, lupeol, lupeol acetate, lupenone, fern-7-en-3-one) and one sterol ( $\beta$-sitosterol) were determined. The presence of these compounds has been shown for the first time in $\mathrm{S}$. veratrifolia. $\alpha$-amyrinone, $\beta$-amyrin, $\beta$-amyrinone, $\psi$-taraxasterol and $\psi$-taraxasterol acetate are new for the genus Scorzonera.
\end{abstract}

Keywords: Scorzonera veratrifolia, triterpenoids, sterol, GC-MS

\section{INTRODUCTION}

The ancient Mediterranean genus Scorzonera L. is a member of the family Asteraceae, subfamily Liguliflorae, tribe Cichorieae. About 160 species of the genus are widely distributed in Eurasia, Central Asia and Africa. Turkey is considered as a diversity centre for the genus with its 52 species, 31 of which are endemic (Altınordu et al. 2015; Coşkunçelebi et al. 2015). Some Scorzonera species have been used as a vegetable (raw or cooked). S. hispanica, S. cretica, S. austriaca, S. mollis, S. suberosa, S. cana, S. semicana and S. papposa are some of the species that are used in the traditional cuisine of various countries (Baytop 1999; Paraschos et al. 2001; Turan et al. 2003; Granica et al. 2015; Mükemre et al. 2016; Xie et al. 2016). Several species of the genus have been utilised as folk remedies. Treatment for pain, fever, rheumatism, wounds, gastrointestinal disorders, snake-bites, carbuncle, mastitis, hepatitis B, malignant stomach neoplasia, dysentery, pulmonary diseases, colds, hypertension, infertility and gout are some of the traditional uses of the genus Scorzonera in several countries including Turkey, Mongolia, China and some European countries (Baytop 1999; Zidorn et al. 2000; Tsevegsuren et al. 2007; Granica et al. 2015; Xie et al. 2016; Yang et al. 2016). Previous phytochemical studies of this genus yielded; dihydroisocoumarins, benzyl phthalides, flavonoids, lignans, neolignans, bibenzyl derivatives, phenolic acid derivatives, kavalactones, sesquiterpenes and triterpenes (Sarı 2012; Granica et al. 2015).

Scorzonera veratrifolia Fenzl is native to East Anatolia and grows on dry rocky hillsides at an altitude of 1600 - 2500 m (Chamberlain 1975). Previously, ethyl acetate fraction obtained from the methanol extract of the plant's roots was studied and two new benzyl phthalides and five phenolic acid derivates were reported (Sarı 2010). Furthermore, it has been reported that the antimicrobial activities of the ethanolic extract, petroleum ether, ethyl acetate and n-butanol fractions of the plant can be used against Staphylococcus aureus, S. epidermidis, Salmonella typhi, Shigella flexneri, Candida albicans (Sarı et al. 2009). This study aimed to investigate the petroleum ether fraction of the methanol extract obtained from the plant's roots. There are no previous records about the chemical composition of the fraction. 


\section{MATERIALS AND METHODS}

\section{Plant material}

Scorzonera veratrifolia was collected from Bitlis, Turkey, at an altitude of $2500 \mathrm{~m}$ in August 2004. A voucher specimen ( F 12 446) was deposited at the Herbarium of the Faculty of Sciences and Letters, Van Yüzüncü Yıl University.

\section{Extraction and fractionation}

The air-dried, ground roots of S. veratrifolia (600 g) were macerated with $\mathrm{MeOH}$ and concentrated under reduced pressure at $45^{\circ} \mathrm{C}$ using a rotary evaporator (Buchi R-200). The methanol extract was dissolved in $\mathrm{MeOH}: \mathrm{H}_{2} \mathrm{O}(1: 2)$ and successively extracted with petroleum ether (PE), ethyl acetate (EtOAc) and $n$-butanol respectively. The PE fraction $(20 \mathrm{~g})$ was subjected to column chromatography (CC) using silica gel (Merck 60, 0.063 $0.200)$ as an adsorbent. The gradient elution was started with $\mathrm{PE}$, continued with the increasing rate of EtOAc and ended with 100\% EtOAc. 82 fractions were provided and grouped based on their Thin Layer Chromatography (TLC, Silica gel, Merck $60 \mathrm{~F}_{254}$ ) findings. Fr 19-21 (7.3 g) was subjected to CC (Silica gel, PE/ $\mathrm{CHCl}_{3}, 80: 20,70: 30,50: 50,0: 100$ ) to afford mixture BCV3 (20 mg, a-amyrin acetate $+\beta$-amyrin acetate + germanicol acetate + lupeol acetate + taraxasterol acetate $+\psi$ - taraxasterol acetate) and mixture BCV6 (20 mg, a-amyrinone + ß-amyrinone + germanicone + lupenone +Fern-7-en-3one). Fr 36-45 (1.3 g) was further separated by CC (silica gel, $\mathrm{CHCl}_{3} / \mathrm{MeOH}, 1: 1$ ) to provide mixture BCV5 (30 mg, a-amyrin
+ B-amyrin + germanicol + lupeol + taraxasterol + $\psi$ - taraxasterol). Fr 52-55 (927 mg) was subjected to CC (silica gel, PE/ AcOEt, $1: 1$ ) and then to prep. TLC (silica gel, PE/AcOEt, 85: 15) to yield pure BCV7 (32.5 mg, ß-sitosterol).

\section{GC-MS and NMR}

Thermo Finnigan Trace GC Ultra (Thermo Electron Corporation) with AS 3000 Autosampler for gas chromatography and Thermo Finnigan Trace DSQ (Thermo Electron Corporation) for mass spectrometry were employed. Details of the method were as follows: Column: ZB 1 MS $0.25 \mu \mathrm{m}$ (30 m x 0.25 mm ID), carrier gas: He, flow rate: $1 \mathrm{~mL} / \mathrm{min}$, injection temperature: $300{ }^{\circ} \mathrm{C}$, column temperature: $65^{\circ} \mathrm{C}$ for 2 minutes, $300^{\circ} \mathrm{C}$ for 20 minutes (increase rate $6^{\circ} \mathrm{C}$ ), injection volume: $2 \mu \mathrm{L}$ and ion source temperature: $200^{\circ} \mathrm{C}$. Full-scan mass spectra were acquired from 1 to $1050 \mathrm{~m} / \mathrm{z}$ at a scan interval of 0.2 in El mode. NMR spectrums were acquired on UNITY INOVA $500 \mathrm{MHz}$ (Varian), in $\mathrm{CDCl}_{3}$.

\section{RESULTS AND DISCUSSION}

The PE fraction of methanol extract obtained from $S$. veratrifolia roots was investigated and six oleanane-type (ß-amyrin, ßamyrin acetate, $ß$-amyrinone, germanicol, germanicol acetate, germanicone), seven ursane-type (a-amyrin, a-amyrin acetate, a-amyrinone, $\psi$ - taraxasterol, $\psi$ - taraxasterol acetate, taraxasterol, taraxasterol acetate), three lupane-type (lupeol, lupeol acetate, lupenone), one fernane-type (Fern-7-en-3-one) triterpenes along with one sterol (B-sitosterol) were determined (Table 1).

Table 1: Triterpenes and $\beta$-sitosterol from the PE fraction of the methanol extract obtained from S. veratrifolia roots determined by GC-MS

\begin{tabular}{|c|c|c|c|c|}
\hline Peak Numbers & Compounds & Retention Time (min) & Molecular Weight & Molecular Formula \\
\hline \multicolumn{5}{|l|}{ Mixture BCV3* } \\
\hline 1 & ß-Amyrin acetate & 45.16 & 468 & $\mathrm{C} 32 \mathrm{H} 52 \mathrm{O} 2$ \\
\hline 2 & Germanicol acetate & 45.34 & 468 & $\mathrm{C} 32 \mathrm{H} 52 \mathrm{O} 2$ \\
\hline 3 & Lupeol acetate $+\alpha$-Amyrin acetate & 46.28 & $468+468$ & $\mathrm{C} 32 \mathrm{H} 52 \mathrm{O} 2$ \\
\hline \multicolumn{5}{|l|}{$\mathrm{C} 32 \mathrm{H} 52 \mathrm{O} 2$} \\
\hline 4 & $\psi$ - Taraxasterol acetate & 47.99 & 468 & $\mathrm{C} 32 \mathrm{H} 52 \mathrm{O} 2$ \\
\hline 5 & Taraxasterol acetate & 48.18 & 468 & $\mathrm{C} 32 \mathrm{H} 52 \mathrm{O} 2$ \\
\hline \multicolumn{5}{|l|}{ Mixture BCV5* } \\
\hline 1 & ß-Amyrin & 43.76 & 426 & $\mathrm{C} 30 \mathrm{H} 500$ \\
\hline 2 & Germanicol & 43.90 & 426 & $\mathrm{C} 30 \mathrm{H} 500$ \\
\hline 3 & Lupeol $+\alpha$-Amyrin & 44.48 & $426+426$ & $\mathrm{C} 30 \mathrm{H} 500$ \\
\hline \multicolumn{5}{|l|}{$\mathrm{C} 30 \mathrm{H} 500$} \\
\hline 4 & $\psi$ - Taraxasterol & 45.92 & 426 & $\mathrm{C} 30 \mathrm{H} 500$ \\
\hline 5 & Taraxasterol & 46.15 & 426 & $\mathrm{C} 30 \mathrm{H} 500$ \\
\hline \multicolumn{5}{|l|}{ Mixture BCV6* } \\
\hline 1 & $\beta$-Amyrinone & 43.50 & 424 & $\mathrm{C} 30 \mathrm{H} 480$ \\
\hline 2 & Germanicone & 43.63 & 424 & $\mathrm{C} 30 \mathrm{H} 480$ \\
\hline 3 & $\alpha$-Amyrinone & 44.17 & 424 & $\mathrm{C} 30 \mathrm{H} 480$ \\
\hline 4 & Fern-7-en-3-one & 44.93 & 424 & $\mathrm{C} 30 \mathrm{H} 480$ \\
\hline 5 & Lupenone & 45.52 & 424 & $\mathrm{C} 30 \mathrm{H} 480$ \\
\hline BCV7* & $\beta$-sitosterol & 43.27 & 414 & $\mathrm{C} 29 \mathrm{H} 500$ \\
\hline
\end{tabular}




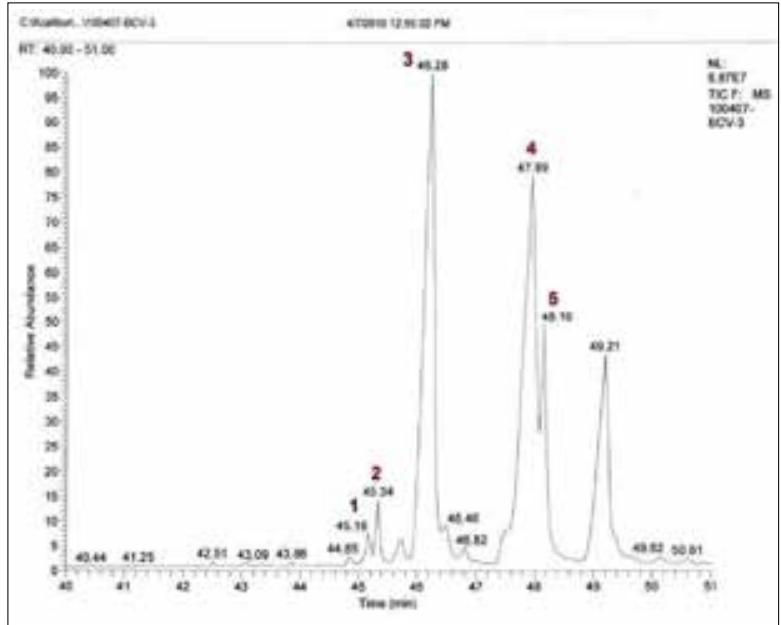

Figure 1. GC chromatogram of mixture BCV3

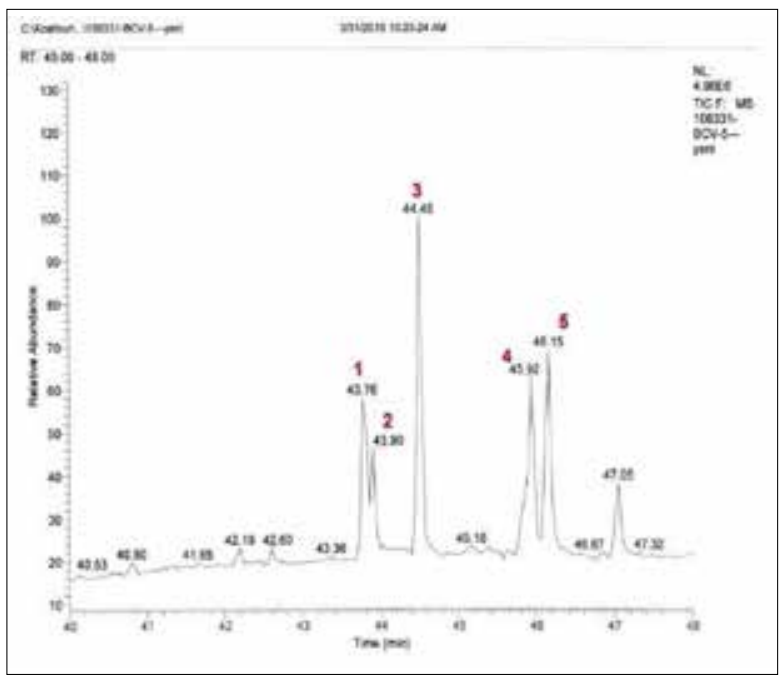

Figure 2. GC chromatogram of mixture BCV5
CC, prep. TLC and GC techniques were used for the separation of the compounds (Figure 1-4). Determination of the compounds was achieved by using GC-MS (Wiley/NIST database) and comparing findings with the literature data (Budzikiewicz et al. 1963; Hooper et al. 1982; Ahmad and Atta ur 1994; Shiojima et al. 1995; Oliveira et al. 2006; Gawrońska-Grzywacz and Krzaczek 2007). Additionally, ${ }^{1} \mathrm{H}$ NMR and ${ }^{13} \mathrm{C}$ NMR techniques were used in the structure elucidation of pure BCV7 (B-sitosterol) (Table 2). The NMR data of the compound was compared with the literature (Pateh et al. 2009).

All compounds were determined for the first time in S. veratrifolia. To the best of our knowledge, a-amyrinone, ß-amyrin, B-amyrinone, $\psi$ - taraxasterol and $\psi$ - taraxasterol acetate are new for the genus Scorzonera. Other triterpenoids were found in several Scorzonera species (Table 3). Particularly, S. veratrifolia showed a similar triterpenoid composition as S. cretica. Also, Bsitosterol has been reported from several Scorzonera species as

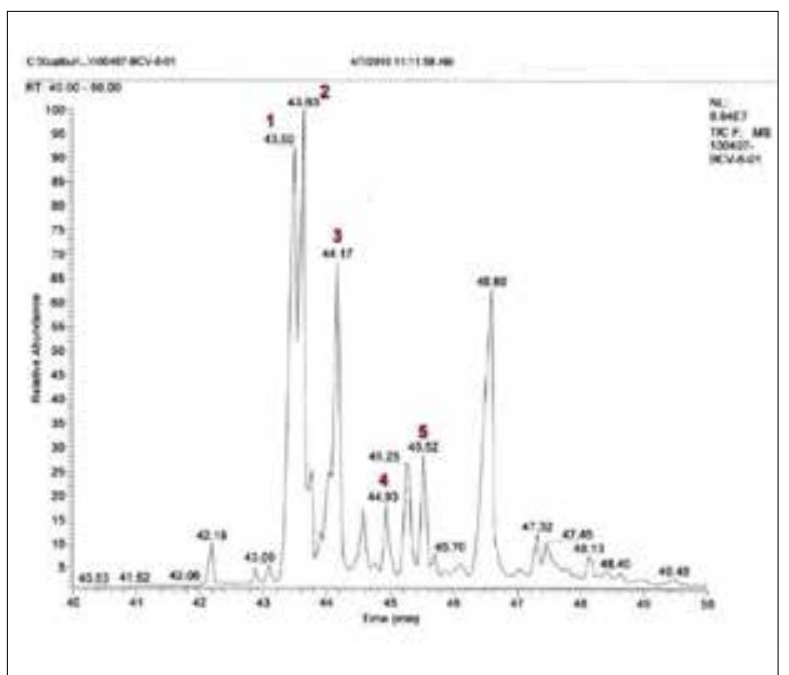

Figure 3. GC chromatogram of mixture BCV6

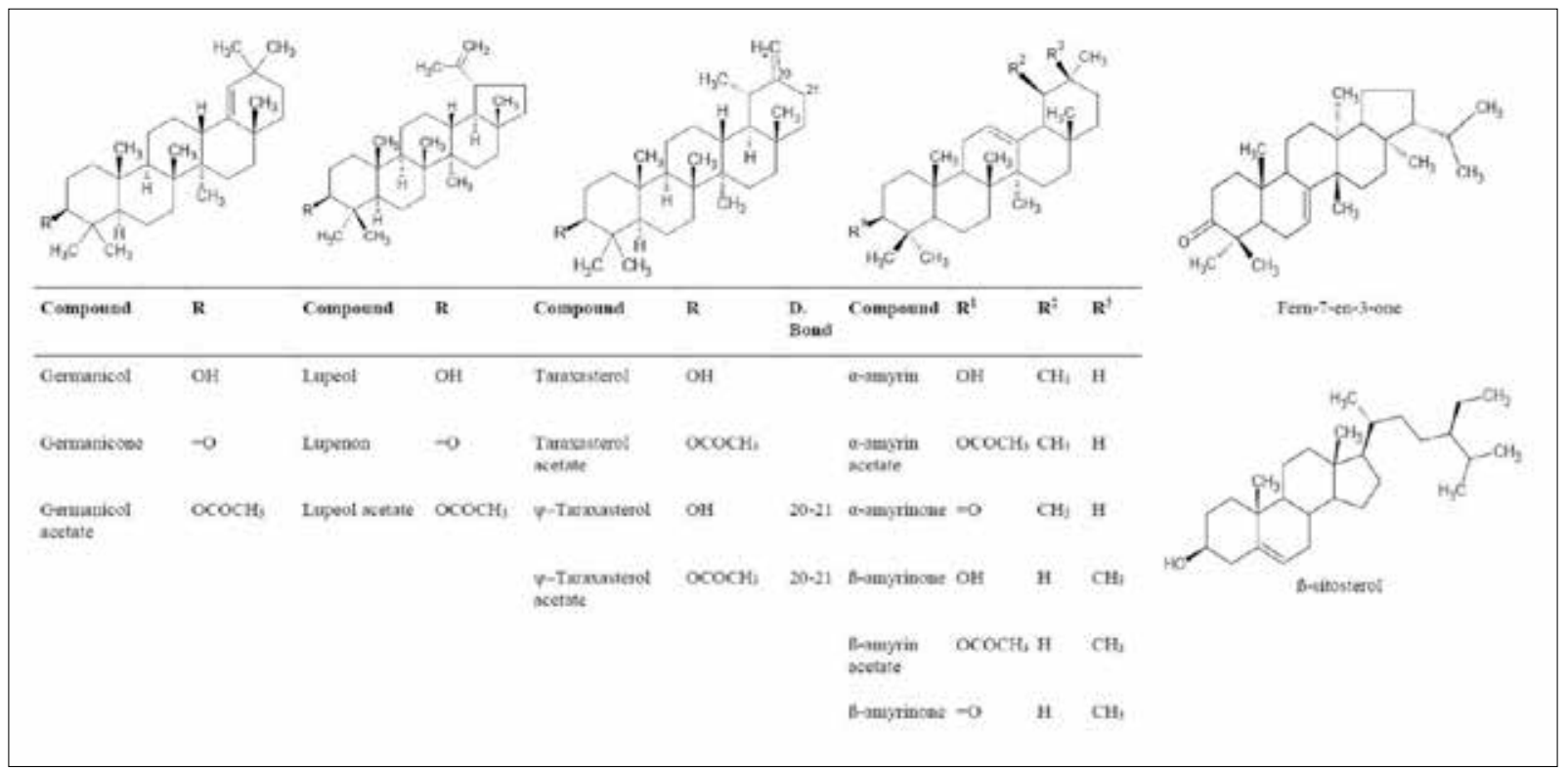

Figure 4. Chemical structures of the compounds determined in the PE fraction of the methanol extract obtained from the roots of Scorzonera veratrifolia 
Table 2: 1H NMR and 13C NMR data of BCV7 (B-sitosterol)

\begin{tabular}{|c|c|c|c|c|c|c|c|c|}
\hline Position & $\delta \mathrm{H}(\mathrm{J}, \mathrm{Hz})$ & $\delta \mathrm{C}$ & Position & $\delta \mathrm{H}(\mathrm{J}, \mathrm{Hz})$ & $\delta \mathrm{C}$ & Position & $\delta \mathrm{H}(\mathrm{J}, \mathrm{Hz})$ & $\delta C$ \\
\hline 1 & & 36.2 & 11 & & 20.0 & 21 & $0.85 d \mid 6.31$ & 17.7 \\
\hline 2 & & 28.7 & 12 & & 38.8 & 22 & & 32.9 \\
\hline 3 & $3.45 \mathrm{~m}$ & 72.0 & 13 & & 41.3 & 23 & & 25.1 \\
\hline 4 & & 41.2 & 14 & & 55.8 & 24 & & 44.8 \\
\hline 5 & & 139.7 & 15 & & 23.3 & 25 & & 27.8 \\
\hline 6 & $5.27 \mathrm{brs}$ & 120.7 & 16 & & 27.2 & 26 & $0.74 \mathrm{~d}(6.8)$ & 18.8 \\
\hline 7 & & 30.6 & 17 & & 54.9 & 27 & $0.77 \mathrm{~d}(6.8)$ & 18.0 \\
\hline 8 & & 30.8 & 18 & $0.61 \mathrm{~s}$ & 10.8 & 28 & 0.77 t (7.8) & 22.0 \\
\hline 9 & & 50.2 & 19 & $0.94 \mathrm{~s}$ & 18.3 & 29 & $0.74 \mathrm{~d}(6.8)$ & 10.9 \\
\hline 10 & & 35.5 & 20 & & 35.1 & & & \\
\hline
\end{tabular}

\section{Table 3: Triterpenoids determined in the genus Scorzonera}

\begin{tabular}{|c|c|}
\hline Species & Triterpenoids \\
\hline S. cretica (Paraschos et al. 2001) & $\begin{array}{l}\text { germanicol, germanicone, germanicol acetate, lupeol, lupenone, lupeol acetate, } \\
\text { taraxasterol, taraxasterol acetate, oleanolic acid, oleanol acetate }\end{array}$ \\
\hline S. tomentosa (Öksüz et al. 1990) & lupeol, lupeol acetate, $\alpha$-amyrin \\
\hline S. aristata (Jehle et al. 2010) & lupeol, magnificol, 3- $\alpha$-hidroxyolean-5-ene \\
\hline S. austriaca (Wu et al. 2011) & 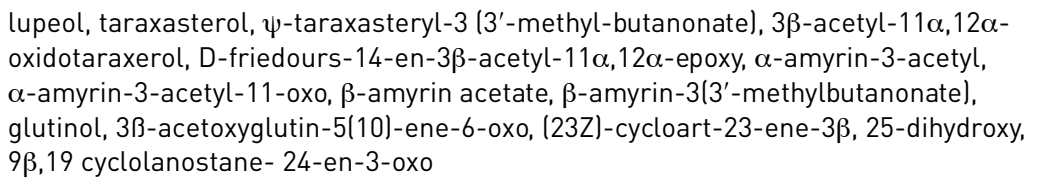 \\
\hline S. columnae (Menichini et al. 1994) & lupeol \\
\hline $\begin{array}{l}\text { S. latifolia (Bahadır et al. 2010; } \\
\text { Acıkara et al. 2012; Acıkara et al. 2014) }\end{array}$ & $\begin{array}{l}\text { fern-7-en-3-one, 3ß-hydroxy-fern-7-ene-6-one-acetate, 3- } \beta \text {-hydroxy-fern-8-ene-7- } \\
\text { one-acetate, fern-7-ene-3-ol, taraxasterol acetate, taraxasterol myristate, olean-12- } \\
\text { ene-11-one-3-acetyl, urs-12-ene-11-one-3-acetyl }\end{array}$ \\
\hline $\begin{array}{l}\text { S. undulata ssp. delicosa } \\
\text { (Harkati et al. 2010) }\end{array}$ & $\beta$-amyrin acetate, methyl oleanate, methyl ursolate \\
\hline $\begin{array}{l}\text { S. undulata ssp. alexandrina } \\
\text { (Benabdelaziz et al. 2014) }\end{array}$ & lupeol, 24-methylenecycloartanol \\
\hline $\begin{array}{l}\text { S. mongolica } \\
\text { (Wang et al. 2007; Wang et al. 2009) }\end{array}$ & $\begin{array}{l}\text { 3ß-tetradecanoyl moradiol, 3-ß-dodecanoyl moradiol, 3ß-tetradecanoyl erythrodiol, } \\
\text { 3-ß-dodecanoyl erythrodiol }\end{array}$ \\
\hline S. divaricata (Yang et al. 2016) & $\begin{array}{l}\text { oleanolic acid, scorzodivaricin B, C, D, 23(Z)-3ß-acetoxy-25-hydroxy-tirucalla-7,23- } \\
\text { diene, 23(Z)-3ß, 25-dihydroxy-tirucalla-7,23-diene, 20(R)-3ß, 21-dihydroxy-24(31)- } \\
\text { methylene-dammarane, 20(R)-3ß-acetoxy-21-hydroxy-24(31)-methylene-dammarane }\end{array}$ \\
\hline $\begin{array}{l}\text { S. hispanica tissue culture } \\
\text { (Tolstikhina et al. 1988) }\end{array}$ & oleanolic acid \\
\hline
\end{tabular}

well (S. tomentosa, S. austriaca, S. columnae, S. latifolia, S. undulata, S. hispanica tissue culture, S. suberosa and S. laciniata) (Tolstikhina et al. 1988; Öksüz et al. 1990; Harkati et al. 2010; Wu et al. 2011; Erden et al. 2013; Acıkara et al. 2014; Benabdelaziz et al. 2014).

GC-MS data of the plants are valuable for setting up chemotaxonomic profiles. However, such studies on the genus Scorzonera are scarce. Moreover, terpenoids are considered to be potential anti-cancer, anti-inflammatory, hepatoprotective, anti-viral agents (Dudhgaonkar et al. 2009; Laszczyk 2009; Thyagarajan et al. 2010; Ding et al. 2011; Gao et al. 2011; Narayan et al. 2011; Dakeng et al. 2012; Ezzat et al. 2012). Thus, further investigations on the terpenoids of the genus Scorzonera are recommended.

\section{Acknowledgements}

The present work was supported by the Research Fund of Istanbul University, Project No: 3506. The authors thank to Dr Fevzi Özgökçe for collecting and identifying the plant material.

\section{REFERENCES}

- Acıkara OB, Çitoglu GS, Dall'Acqua S, Smejkal K, Cvacka J, Zemlicka M (2012). A new triterpene from Scorzonera latifolia (Fisch. and Mey.) DC. Nat Prod Res 26: 1892-1897. [CrossRef]

- Acıkara ÖB, Çitoğlu GS, Acqua SD, Cvacka J, Zemlicka M, Smejkal K (2014). Bioassay-guided isolation of the antinociceptive compounds motiol and ß-sitosterol from Scorzonera latifolia root extract. Pharmazie 69: 711-714. 
- $\quad$ Ahmad VU, Atta ur R (1994). Handbook of natural products data. Volume 2: pentacyclic triterpenoids. Elsevier Science, Amsterdam.

- Altınordu F, Martin E, Makbul S, Coşkunçelebi K, Gültepe M (2015). Cytogenetic studies on some Scorzonera L. s.l. (Asteraceae) taxa from Turkey. Turk J Bot 39: 429-438. [CrossRef]

- Bahadır O, Çitoglu GS, Smejkal K, Dall'Acqua S, Özbek H, Cvacka J, Zemlicka M (2010). Analgesic compounds from Scorzonera latifolia (Fisch. and Mey.) DC. J Ethnopharmacol 131: 83-87. [CrossRef]

- Baytop T (1999). Türkiye'de Bitkilerle Tedavi Geçmişte ve Bugün (Therapy with medicinal plants in Turkey). Nobel Tıp, İstanbul.

- Benabdelaziz I, Haba H, Lavaud C, Benkhaled M (2014). Triterpenoids and flavonoid from Scorzonera undulata ssp. alexandrina. Int J Chem Biol Sci 5: 1-5.

- Budzikiewicz H, Wilson JM, Djerassi C (1963). Mass Spectrometry in Structural and Stereochemical Problems. XXXII.1 Pentacyclic Triterpenes. J Am Chem Soc 85: 3688-3699. [CrossRef]

- Chamberlain DF (1975). Scorzonera L. In: PH Davis, VA Matthews, FK Kupicha, BS Parris (eds) Flora of Turkey and the East Aegean Islands, V, Edinburgh University Press, Edinburgh, 632-657.

- Coşkunçelebi K, Makbul S, Gültepe M, Okur S, Güzel ME (2015). A conspectus of Scorzonera s.l. in Turkey. Turk J Bot 39: 76-87. [CrossRef]

- Dakeng S, Duangmano S, Jiratchariyakul W, U-Pratya Y, Bögler O, Patmasiriwat P (2012). Inhibition of Wnt signaling by cucurbitacin $B$ in breast cancer cells: Reduction of Wnt associated proteins and reduced translocation of galectin-3-mediated $\beta$-catenin to the nucleus. J Cell Biochem 113: 49-60. [CrossRef]

- Ding N, Yamashita U, Matsuoka H, Sugiura T, Tsukada J, Noguchi J, Yoshida Y (2011). Apoptosis induction through proteasome inhibitory activity of cucurbitacin D in human T-cell leukemia. Cancer 117: 2735-2746. [CrossRef]

- Dudhgaonkar S, Thyagarajan A, Sliva D (2009). Suppression of the inflammatory response by triterpenes isolated from the mushroom Ganoderma lucidum. Int J Immunopharmacol 9: 1272-1280. [CrossRef]

- $\quad$ Erden Y, Kırbağ S, Yılmaz Ö (2013). Phytochemical composition and antioxidant activity of some Scorzonera species. Proc Natl Acad Sci, India, Sect B 83: 271-276.

- Ezzat SM, Abdallah HM, Fawzy GA, El-Maraghy SA (2012). Hepatoprotective constituents of Torilis radiata Moench (Apiaceae). Nat Prod Res 26: 282-285. [CrossRef]

- Gao Y, Zhang R, Zhang J, Gao S, Gao W, Zhang H, Wang H, Han $B$ (2011). Study of the extraction process and in vivo inhibitory effect of Ganoderma triterpenes in oral mucosa cancer. Molecules 16: 5315. [CrossRef]

- Gawrońska-Grzywacz M, KrzaczekT (2007). Identification and determination of triterpenoids in Hieracium pilosella L. J Sep Sci $\mathbf{3 0}$ 746-750. [CrossRef]

- Granica S, Lohwasser U, Johrer K, Zidorn C (2015). Qualitative and quantitative analyses of secondary metabolites in aerial and subaerial of Scorzonera hispanica L. (Black Salsify). Food Chem 173 321-331. [CrossRef]

- Harkati B, Akkal S, Bayat C, Laouer H, Franca MGD (2010). Secondary metabolites from Scorzonera undulata ssp. deliciosa (Guss.) Maire (Asteracae) and their antioxidant activities. Rec Nat Prod 4: 171-175.

- Hooper SN, Chandler RF, Lewis E, Jamieson WD (1982). Simultaneous determination of Sonchus arvensis $L$. triterpenes by gas chromatography-mass spectrometry. Lipids 17: 60-63. [CrossRef]

- Jehle M, Bano J, Ellmerer EP, Zidorn C (2010). Natural products from Scorzonera aristata (Asteraceae). Nat Prod Commun 5: 725-727.

- Laszczyk MN (2009). Pentacyclic triterpenes of the lupane, oleanane and ursane group as tools in cancer therapy. Planta Med 75: 1549-1560. [CrossRef]

- Menichini F, Statti G, Delle Monache F (1994). Flavonoid glycosides from Scorzonera columnae. Fitoterapia 65: 555-556.
Mükemre M, Behçet L, Çakılcıoğlu U (2016). Survey of wild food plants for human consumption in villages of Çatak (Van-Turkey). Indian J Tradit Knowle 15: 183-191.

- $\quad$ Narayan V, Kodihalli RC, Chiaro C, Cary D, Aggarwal BB, Henderson AJ, Prabhu KS (2011). Celastrol inhibits tat-mediated Human Immunodeficiency Virus (HIV) transcription and replication. J Mol Biol 410: 972-983. [CrossRef]

- $\quad$ Oliveira P, Turatti I, Camilo Rodrigues de Oliveira D (2006). Comparative analysis of triterpenoids from Mikania cordifolia collected from four different locations. Braz J Pharm Sci 42: 547-552. [CrossRef]

- $\quad$ Öksüz S, Gören N, Ulubelen A (1990). Terpenoids from Scorzonera tomentosa. Fitoterapia 61: 92-93.

Paraschos S, Magiatis P, Kalpoutzakis E, Harvala C, Skaltsounis AL (2001). Three new dihydroisocoumarins from the Greek endemic species Scorzonera cretica. J Nat Prod 64: 1585-1587. [CrossRef]

Pateh UU, K. HA, Garba M, Iliya I, Sule IM, Abubakar MS, A.A. A (2009). Isolation of stigmasterol, ß-sitosterol and 2-hydroxyhexadecanoic acid methyl ester from the rhizomes of Stylochiton lancifolius Pyer and Kotchy (Araceae). Nig Journ Pharm Sci 8: 19-25.

- Sarı A (2010). Two new 3-benzylphthalides from Scorzonera veratrifolia Fenzl. Nat Prod Res 24: 56-62. [CrossRef]

- Sarı A (2012). Phenolic compounds from Scorzonera latifolia (Fisch. \& Mey.) DC. Nat Prod Res 26: 50-55. [CrossRef]

- Sarı A, Özbek B, Özgökçe F (2009). Antimicrobial activities of two Scorzonera species growing in Turkey. Asian J Chem 21: 47854788.

- $\quad$ Shiojima K, Masuda K, Suzuki H, Lin T, Ooishi Y, Ageta H (1995). Composite constituents : forty-two triterpenoids including eight novel compounds isolated from Picris hieracioides subsp. japonica. Chem Pharm Bull 43: 1634-1639. [CrossRef]

- Thyagarajan A, Jedinak A, Nguyen H, Terry C, Baldridge LA, Jiang J, Sliva D (2010). Triterpenes from Ganoderma lucidum induce autophagy in colon cancer through the inhibition of p38 mitogenactivated kinase (p38 MAPK). Nutrition and Cancer 62: 630-640. [CrossRef]

- Tolstikhina VV, Bryanskii OV, Syrchina Al, Semenov AA (1988). Chemical composition of a culture of tissue of Scorzonera hispanica. Chem Nat Compd 24: 655-655. [CrossRef]

- Tsevegsuren N, Edrada R, Lin W, Ebel R, Torre C, Ortlepp S, Wray $V$, Proksch P (2007). Biologically active natural products from Mongolian medicinal plants Scorzonera divaricata and Scorzonera pseudodivaricata. J Nat Prod 70: 962-967. [CrossRef]

- Turan M, Kordali S, Zengin H, Dursun A, Sezen Y (2003). Macro and micro mineral content of some wild edible leaves consumed in eastern Anatolia. Acta Agric Scand Sect B Soil Plant Sci 53: 129-137. [CrossRef]

Wang B, Li G, Guan H, Yang L, Tong G (2009). A new erythrodiol triterpene fatty ester from Scorzonera mongolica. Acta Pharm Sin 44: 1258-1261.

- Wang B, Li GQ, Qiu PJ, Guan HS (2007). Two new olean-type triterpene fatty esters from Scorzonera mongolica. Chin Chem Lett 18 : 708-710. [CrossRef]

- Wu QX, Su YB, Zhu Y (2011). Triterpenes and steroids from the roots of Scorzonera austriaca. Fitoterapia 82: 493-496. [CrossRef]

- Xie Y, Guo QS, Wang GS (2016). Flavonoid glycosides and their derivatives from the herbs of Scorzonera austriaca Wild. Molecules 21: 803. [CrossRef]

- $\quad$ Yang YJ, Yao J, Jin XJ, Shi ZN, Shen TF, Fang JG, Yao XJ, Zhu Y (2016). Sesquiterpenoids and tirucallane triterpenoids from the roots of Scorzonera divaricata. Phytochemistry 124: 86-98. [CrossRef]

Zidorn C, Ellmerer-Müller EP, Stuppner H (2000). Tyrolobibenzyls Novel secondery metabolites from Scorzonera humilis. Helv Chim Acta 83: 2920-2925. [CrossRef] 